\title{
A Simple Percutaneous Technique to Reduce Valgus-Impacted Femoral Neck Fractures
}

\author{
Byung-Ho Yoon, MD, Yoon Seok Kim, MD, Kyung-Hoi Koo, MD* \\ Department of Orthopedic Surgery, Seoul Paik Hospital, Inje University College of Medicine, Seoul, \\ * Department of Orthopedic Surgery, Seoul National University Bundang Hospital, Seoul National University College of Medicine, Seongnam, Korea
}

The mainstream surgery for valgus-impacted femoral neck fractures (FNFs) is closed reduction and internal fixation under fluoroscopy. However, femoral neck shortening and anterior femoroacetabular impingement are common complications in healed valgusimpacted FNFs after in situ fixation. Some methods have been reported to prevent complications, but these techniques require the use of a transfixing guide wire that passes through the femoral head, which may cause articular cartilage damage. We introduce a simple reduction technique using one Steinmann pin (S-pin) for valgus-impacted FNFs without any interference of the femur head. A S-pin was placed percutaneously at the inferomedial margin of the fracture, and reduction was achieved by applying manual valgus force along the fracture line. By restoring the anatomical alignment, we confirmed the maximum contact area of the fracture and connection of the medial cortical buttress, so the risk of nonunion was also minimized.

Keywords: Femur neck, Fracture fixation, Internal, Coxa vara

A femoral neck fracture (FNF) is a common hip fracture. There are two strategies for surgical treatment, depending on the fracture pattern and patient age: arthroplasty and internal fixation. Most older individuals with displaced FNFs are treated with arthroplasty. However, the mainstream surgery for a stable FNF is closed reduction and internal fixation under fluoroscopy. ${ }^{1,2}$ Most surgeons agree that the valgus-impacted FNF is a stable fracture, and it is usually treated with in situ fixation. ${ }^{1-3)}$ However, in some cases, it is associated with a poor prognosis due to high femoral neck deformity, low quality of life, and a high revision rate. ${ }^{4,5)}$

Femoral neck shortening is common and causes functional problems after internal fixation of a valgusimpacted FNF. ${ }^{2)}$ The frequency of significant femoral neck shortening after screw fixation of FNFs is roughly $30 \%{ }^{2,4)}$

Received November 8, 2019; Accepted November 22, 2019

Correspondence to: Kyung-Hoi Koo, MD

Department of Orthopedic Surgery, Seoul National University Bundang Hospital, Seoul National University College of Medicine, 82 Gumi-ro 173beon-gil, Bundang-gu, Seongnam 13620, Korea

Tel: +82-31-787-7204, Fax: +82-31-787-4056

E-mail:khkoo@snu.ac.kr
A significant decrease in abductor strength was observed in patients with a valgus-healed femoral neck due to a decrease in the moment arm. ${ }^{6}$ In addition, many studies verified that a healed valgus-impacted FNF can cause anterior femoroacetabular impingement (FAI) because of a large bump on the anterolateral side of the femoral headneck transition that causes a cam lesion on the femoral neck and leads to progressive hip arthrosis. ${ }^{5)}$

Steinmann pins (S-pins) are a thin metal rod commonly used in orthopedic procedures to hold large bone fractures together or control the reduction of the fracture. Here, we describe a simple closed reduction technique using one S-pin for anatomical restoration of the femoral neck in the surgical treatment of the valgus-impacted FNF.

\section{TECHNIQUE}

The patient was placed in a supine position on a fracture traction table. The injured lower extremity was fixed to the table, and the contralateral leg was placed in the lithotomy position with flexion and abduction of the hip, which facilitates the positioning of the fluoroscope and allows anteroposterior (AP) and lateral radiographic screening of the injured hip. 
Yoon et al. Percutaneous Technique to Reduce Valgus Impacted Femoral Neck Fracture

Clinics in Orthopedic Surgery • Vol. 12, No. 2, $2020 \bullet$ www.ecios.org

An important draping tip is that the medial aspect of the groin area should be sufficiently exposed to allow medial positioning of the S-pin in the fracture site. The perineal post was placed in a medialized position toward the operative site, and the medial aspect of the groin was exposed after the final sterile draping. Then, minimal traction was applied to the leg, and the anatomical landmarks of the femoral head, neck, and fracture site were marked under fluoroscopic guidance. A stab incision was made at the center of the femoral neck in line with the fracture line. The in-out direction of the S-pin from medial to lateral avoids injury to the blood vessels and nerves and facilitates the application of valgus force to the fracture (Fig. 1).

A S-pin was placed percutaneously through the stab incision into the femoral neck under the guidance of Carm in the AP direction, and the position was confirmed fluoroscopically. The S-pin was determined to be 2.8-3.6 $\mathrm{mm}$ depending on the size of the patient. We placed the $S$-pin under the inferomedial margin of the fracture line, and when the pin was confirmed to be at the correct position by fluoroscopy, a manual varus force was applied to the femoral neck along the fracture line to reduce the angulation (Fig. 2). Once the valgus-impacted FNF was disimpacted into the anatomical position and the cortical continuity of the medical cortex was recovered, reduction was checked fluoroscopically with the reduction force maintained, and the central guide pin was inserted percutaneously at sufficient depth to reach the subchondral bone (Fig. 3A). After the guide pin was inserted properly, as observed on the AP view, the S-pin for reduction was removed, and the reduction status was checked on the lateral view (Fig. 3B). Additional guide pin or provisional anti-rotation pin was inserted depending on the device used (Fig. 3C). The surgical exposure can vary depending on which surgical technique or fixation device is used.
We used multiple full-threaded cannulated screws (Stryker Orthopaedics, Mahwah, NJ, USA) and the femoral neck system (DePuy Synthes, West Chester, PA, USA), and all procedures were carried out percutaneously with only a short linear incision made just below the vastus lateralis ridge of the greater trochanter (Fig. 4). After surgery, patients were allowed to get out of bed on the second postoperative day and to bear partial weight as tolerated with the aid of a walker for 4 weeks postoperatively; they switched to using a single cane with full weight-bearing in the second 4 weeks.

We administered this surgical technique in five patients (one man and four women) ranging from 36 to 61 years in age. The total surgical time was 35 to 50 minutes. All cases were followed-up for 1-3.1 years (average, 2.1 years), and there were no long-term complications such as nonunion, osteonecrosis of the femoral head, femoral neck shortening, and FAI syndrome.

\section{Ethics Approval and Informed Consent}

The protocol of this study was reviewed and approved by the Institutional Review Board of Seoul Paik Hospital (IRB No. PAIK 2019-10-008-001).

\section{DISCUSSION}

Several articles proposed the repositioning of valgus impaction, but these techniques require the use of a transfixing guide wire that passes through the femoral head. ${ }^{7,8)}$ Our technique does not require additional pin insertion or rotational motion of the femoral head, and it reduces the risk of cartilage and nutritional vessel injury. We expect that this simple reduction technique will increase the bone contact area by restoring the femoral neck alignment. ${ }^{2,6)}$

Previous reports suggested that the optimal posi-
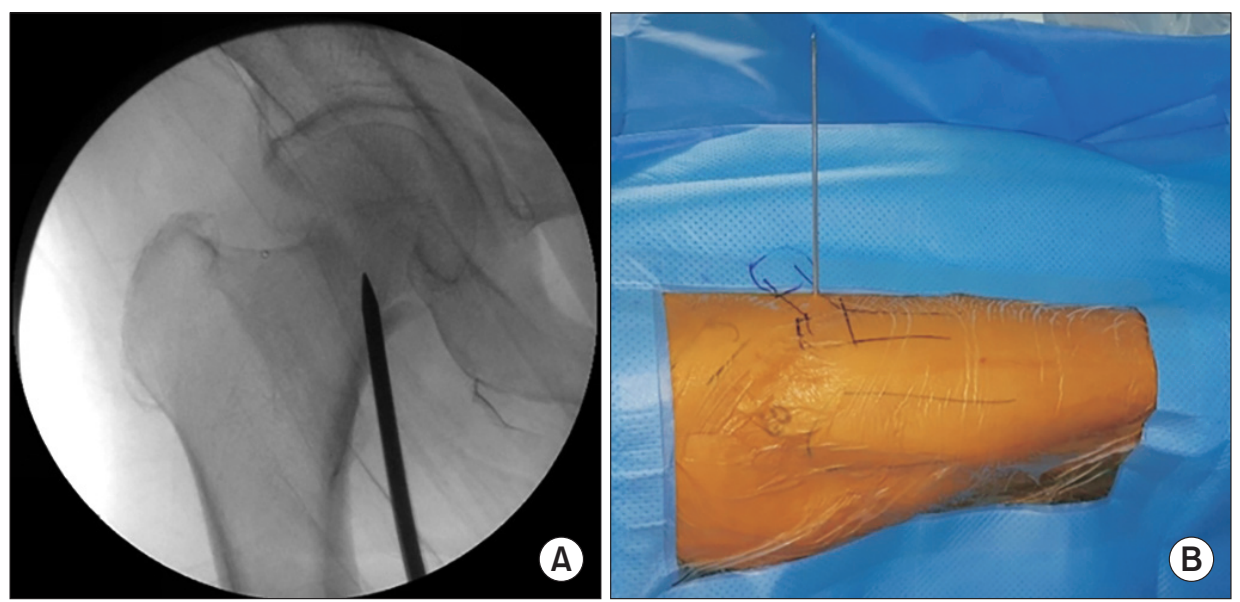

Fig. 1. Supine positioning of the patient on the fracture table and draping of the right hip. (A) The Steinmann pin (S-pin) was placed percutaneously through the stab incision in an in-out direction under the guidance of $\mathrm{C}$-arm. (B) The S-pin was placed in the center of the fracture and its position was confirmed fluoroscopically. 
Yoon et al. Percutaneous Technique to Reduce Valgus Impacted Femoral Neck Fracture

Clinics in Orthopedic Surgery • Vol. 12, No. 2, $2020 \bullet$ www.ecios.org
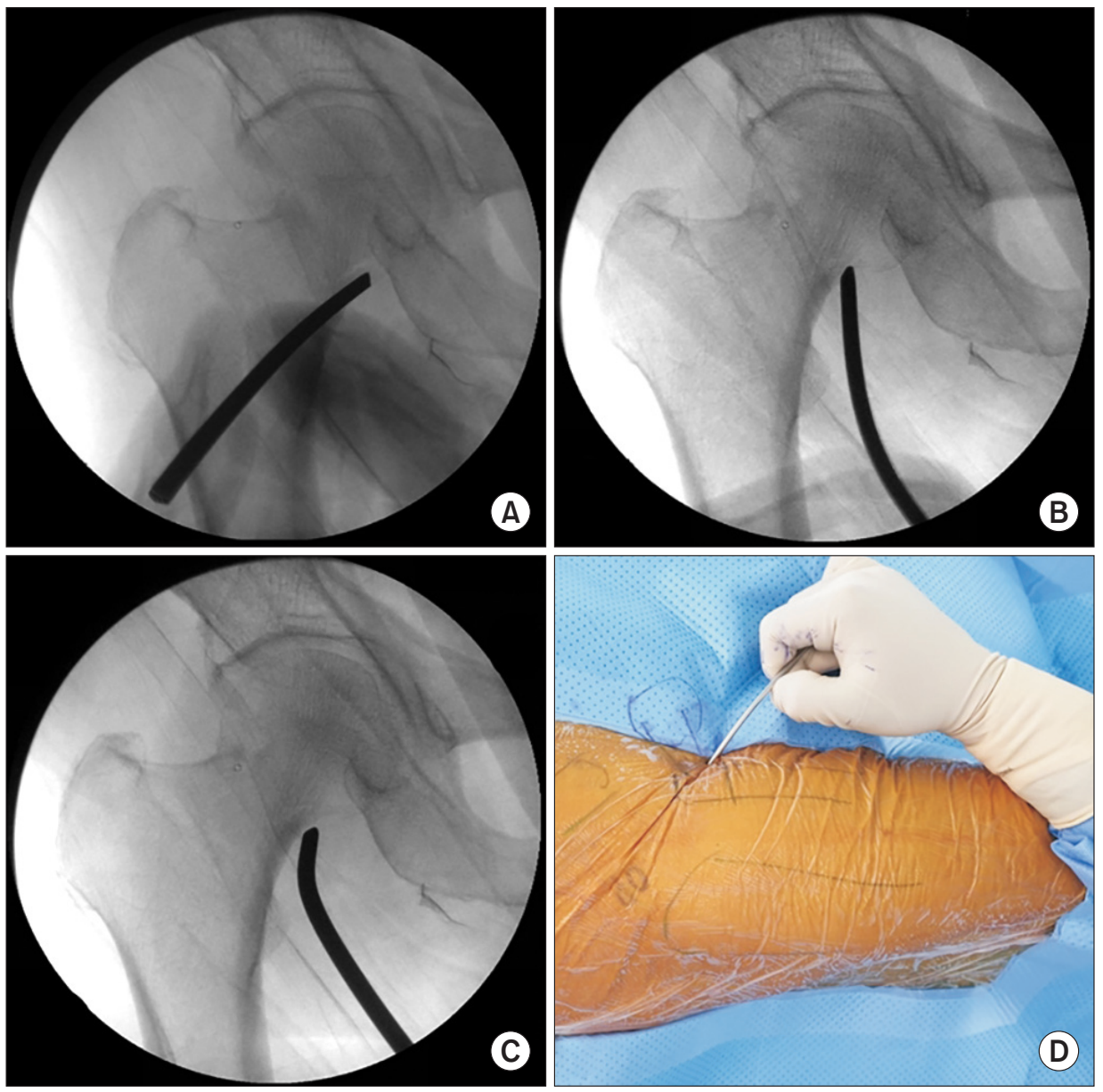

Fig. 2. The Steinmann pin (S-pin) was placed below the inferomedial margin of the fracture line, and if the pin was confirmed to be at the right position by fluoroscopy $(A)$, a pull-up force was applied to the margin of the fracture by pushing the S-pin (B). (C, D) Once the fracture fragment was disengaged, the maximum possible force was applied to the fracture to restore the anatomical alignment.
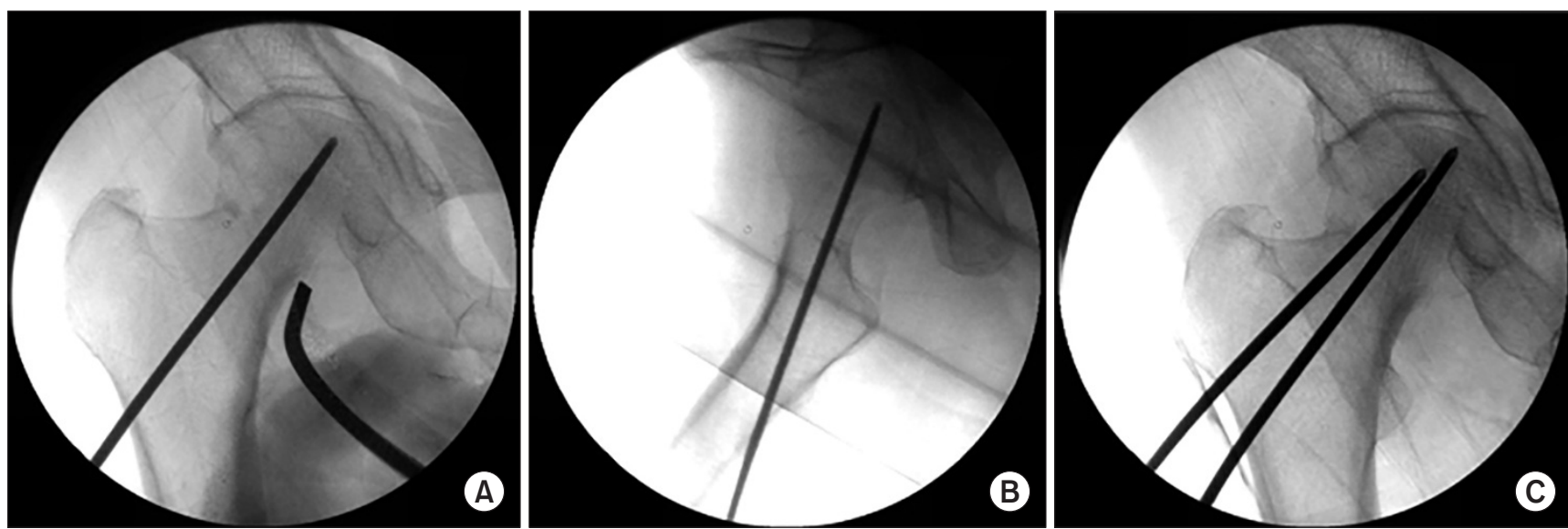

Fig. 3. (A) After the anatomical alignment was restored, a central guide pin was inserted percutaneously and deeply enough to reach the subchondral bone. (B) If the guide pin was inserted properly on the anteroposterior view, the Steinmann pin was removed for reduction, and the reduction status was checked on the lateral view. (C) Additional guide pin or provisional anti-rotation pin was inserted depending on the fixation device.

tioning of screws over a wide area would reduce the risk of nonunion after multiple-screw fixation. ${ }^{1)}$ Valgus deformity in the femoral neck leads to a decrease in the femoral neck dimension, which reduces the contact area and space for screw insertion. This hinders the optimal positioning of screws. By restoring anatomical alignment, our technique allows maximum screw-to-screw clearance and decreases the incidence of nonunion. 
Yoon et al. Percutaneous Technique to Reduce Valgus Impacted Femoral Neck Fracture

Clinics in Orthopedic Surgery • Vol. 12, No. 2, $2020 \bullet$ www.ecios.org
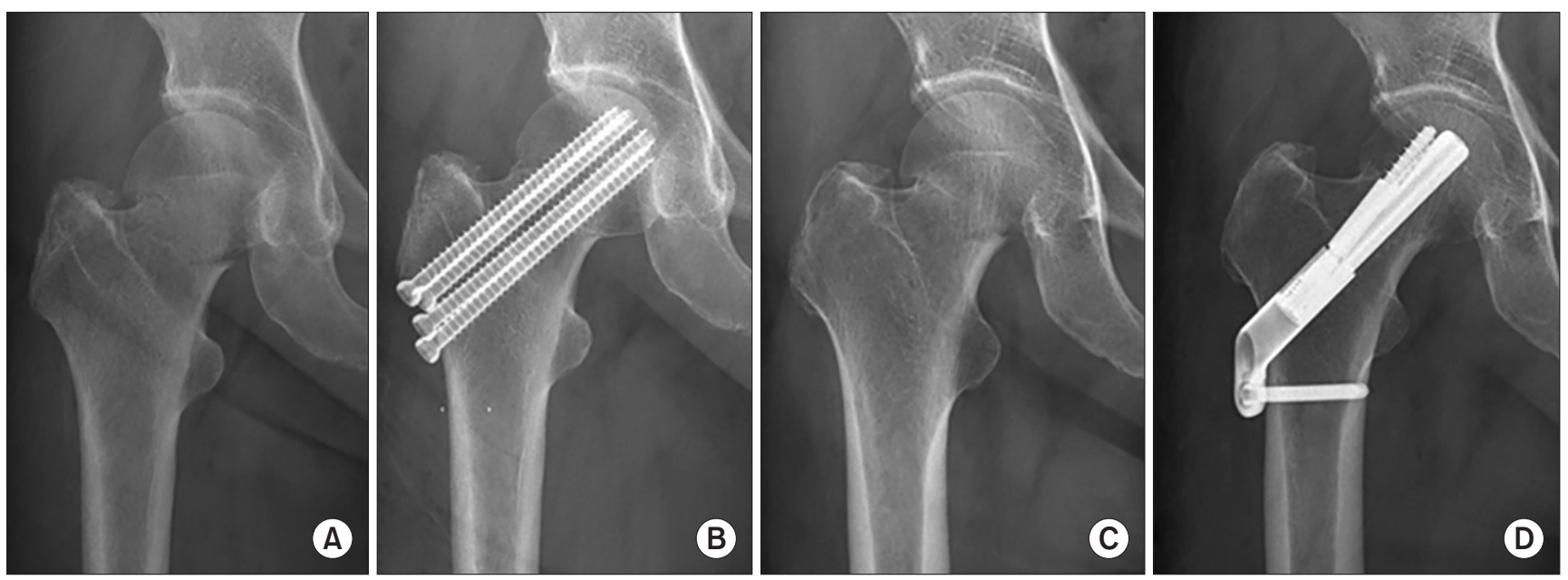

Fig. 4. (A) Pelvic anteroposterior $X$-ray demonstrating the valgus-impacted fracture of the neck of the right femur in a 45-year-old female patient. (B) At 12 months postoperatively, the radiograph showed complete union of the fracture without any deformity. (C) Pelvic anteroposterior $X$-ray demonstrating the valgus-impacted fracture of the neck of the right femur in a 61-year-old female patient. (D) At 9 months postoperatively, the radiograph showed complete union of the fracture without any deformity.

Many patients may not be suitable candidates for reoperation after internal fixation of FNF even when they experience a range of suboptimal outcomes. ${ }^{5}$ There are several studies on the incidence and risk factors of femoral neck shortening after FNFs. It is suggested that nonparallel screw fixation or the use of length-stable implants will reduce the incidence of femoral neck shortening and collapse after FNFs. ${ }^{3,4)}$ We used full-threaded screws to prevent femoral neck shortening after complete reduction using cannulated screws. However, sufficient fracture reduction is more important than the optimal positioning of the implants.

Anterior FAI is another cause of ongoing hip pain and dysfunction in patients with healed FNFs after surgical treatment. According to Strehl A, FAI may cause chronic pain in patients with a healed FNF in the absence of femoral head necrosis. ${ }^{9)}$ The overall prevalence of radiographic FAI was $75 \%$ in patients with a healed FNF that was treated with reduction and internal fixation. ${ }^{10)} \mathrm{Al}-$ though it is difficult to derive strong conclusions regarding the correlation between FAI and chronic pain, anatomical fracture reduction should be performed to improve the patient outcome. ${ }^{9,10)}$

There are some limitations to our technique. First, a high posterior tilt angle in an FNF is an independent risk factor of nonunion, but it is difficult to reduce the posterior tilt with our technique. Thus, surgeons should apply this technique with caution, especially in cases of a highly deformed posterior valgus-impacted FNF. Second, a patient-reported outcomes questionnaire is necessary to fully evaluate the outcomes from the patient's perspective.

We introduced a simple reduction technique for valgus impacted FNFs, which increases the contact area of the fracture and connects the medial cortical buttress. The important advantage of this technique is that it decreases the possibility of femoral neck shortening and FAI without any interference of the femoral head.

\section{CONFLICT OF INTEREST}

No potential conflict of interest relevant to this article was reported.

\section{REFERENCES}

1. Nyholm AM, Palm H, Sandholdt H, Troelsen A, Gromov K; Danish Fracture Database Collaborators. Osteosynthesis with parallel implants in the treatment of femoral neck fractures: minimal effect of implant position on risk of reoperation. J Bone Joint Surg Am. 2018;100(19):1682-90.
2. Zlowodzki M, Ayeni O, Petrisor BA, Bhandari M. Femoral neck shortening after fracture fixation with multiple cancellous screws: incidence and effect on function. J Trauma. 2008;64(1):163-9.

3. Yoon BH, Kwon MS. Atraumatic bilateral fracture of the 
Yoon et al. Percutaneous Technique to Reduce Valgus Impacted Femoral Neck Fracture

Clinics in Orthopedic Surgery • Vol. 12, No. 2, $2020 \bullet$ www.ecios.org

femoral neck in young male patient with suspected osteomalacia. J Bone Metab. 2017;24(3):197-200.

4. Felton J, Slobogean GP, Jackson SS, et al. Femoral neck shortening after hip fracture fixation is associated with inferior hip function: results from the FAITH trial. J Orthop Trauma. 2019;33(10):487-96.

5. Leonardsson O, Rolfson O, Hommel A, Garellick G, Akesson K, Rogmark C. Patient-reported outcome after displaced femoral neck fracture: a national survey of 4467 patients. J Bone Joint Surg Am. 2013;95(18):1693-9.

6. Noda M, Saegusa Y, Takahashi M, et al. Diminished abductor muscular strength in patients with valgus-impacted femoral neck fractures treated by internal fixation: clinical study and biomechanical considerations. J Orthop Surg (Hong Kong). 2017;25(2):2309499017716070.
7. Noda M, Takahashi M, Nukuto $K$, et al. Innovative technique of minimally invasive closed reduction for impacted femoral neck fractures (MICRIF). J Orthop Surg (Hong Kong). 2019;27(1):2309499019832418.

8. Mahajan RH, Kumar S, Mishra B. Technique for gentle accurate reproducible closed reduction of intracapsular fracture of neck of femur. Injury. 2017;48(3):789-90.

9. Strehl A, Ganz R. Anterior femoroacetabular impingement after healed femoral neck fractures. Unfallchirurg. 2005;108(4):263-73.

10. Wendt MC, Cass JR, Trousdale RR. Incidence of radiographic cam-type impingement in young patients $(<50)$ after femoral neck fracture treated with reduction and internal fixation. HSS J. 2013;9(2):113-7. 